\title{
Chronischer Husten bei Kindern kann eine lange Spurensuche erfordern
}

\author{
Bei Kindern mit chronischem, länger als vier Wochen anhaltenden Husten ist die Spurensuche mitunter \\ schwierig. Wegweisend sind die Qualität des Hustens (bellend, röhrend, produktiv, trocken), der Auskulta- \\ tionsbefund und das Timing des Hustens, zum Beispiel nachts oder unter Belastung.
}

Anamnese und Auskultation sind zunächst die wichtigsten Instrumente bei der Differenzialdiagnose. Ist ein Giemen oder Stridor zu hören, deutet dies auf eine Obstruktion hin, sagte Prof. Jürgen Seidenberg aus Oldenburg. Rasselgeräusche können durch eine Bronchiolitis oder Alveolitis ausgelöst werden, eine Bronchophonie ist ein möglicher Hinweis auf eine Pneumonie und Seitendifferenz für einen Erguss, eine Atelektase oder Überblähung. Die Auskultation kann auch den Verdacht einer Fremdkörperaspiration verstärken, die zu 70\% mit Husten einhergeht. Bei etwa der Hälfte der Kinder mit Fremdkörperaspiration sind die Atemgeräusche abgeschwächt oder es ist beim Atmen ein Pfeifen zu hören, so Seidenberg.

\section{Wie gehustet wird, ist wichtig}

Die Qualität des Hustens liefert wichtige Hinweise auf die Ursache. Bei Kleinkindern liegt häufig ein feucht-schleimiger Husten vor, der meist nicht produktiv ist, weil der Schleim runtergeschluckt wird, sagte der Pädiater. Diese Kinder leiden häufig an einer protrahierten bakteriellen Bronchitis, ausgelöst überwiegend durch eine Infektion mit Haemophilus influenzae, Streptococcus pneumoniae oder Moraxella catarrhalis. Betroffen sind nach Erfahrung von Seidenberg meist Kinder unter 2 Jahren, die häufig länger als ein Jahr Husten hätten. Viele Betroffene würden mit Anti-Asthmatika behandelt. Für eine Symptomfreiheit sind oft 2, selten mehr antibiotische Zyklen nötig. Bei Kindern mit feucht-schleimigem Husten sollte aber auch an Immundefekte, Mukoviszidose und chronische Sinusitis aufgrund vergrößerter Adenoide, auch upper airway cough syndrom (UACS) genannt, gedacht werden. Zu den Therapieoptionen bei UACS zählen nasale Steroide, bei V.a. eine allergische Anamnese auch Antihistaminika und Leukotrienantagonisten.

Auch ein Stakkatohusten wird häufig durch eine Infektion ausgelöst, etwa durch Chlamydien oder durch RS-Viren. Ist am Ende der Ausatmung ein Keuchen zu hören, sollte auch an Pertussis gedacht werden, so Seidenberg. Er erinnerte an eine britische Studie bei 170 Schulkindern mit persistierendem Husten. Bei mehr als zwei Drittel der Patienten ergaben sich serologische Hinweise auf eine akute Pertussisimpfung, obwohl $86 \%$ der Kinder eine Grundimmunsierung erhalten hatten (Harnden A, BMJ 2006; 333(7560):174-7).

Bei trockenem Husten, vor allem unter körperlicher Belastung und nachts, besteht dringender Verdacht auf ein coughvariant-Asthma, sagte Seidenberg. Als Risikofaktoren für ein meist allergisches Asthma im Schulkindalter nannte er positive Familienanamnese, atopisches Ekzem im Säuglingsalter, frühe Sensibilisierung (Hühnereiweiß, Hausstaubmilbe, Tierhaare), 3 oder mehr obstruktive Bronchitiden, Giemen im infektfreien Intervall und vermehrte Eosinophile. Ist die Diagnose unklar, kann auch ein Therapieversuch mit inhalativem Kortikosteroid (plus
LABA) über 2-3 Wochen erfolgen, berichtete Seidenberg. Höre der Husten auf, liege Asthma bzw. eine asthmaähnliche Erkrankung vor, sonst müsse weitergefahndet werden. Auch bei anhaltendem produktivem Husten kann ein diagnostischer Therapieversuch mit Antibiotika erfolgen. Sei der Husten in 2-3 Wochen vorbei, lag eine protrahierte Bronchitis vor, sonst sei eine weitere Diagnostik erforderlich.

Bei unklarem röhrendem Husten, „wie eine kanadische Wildgans“, so der Pädiater, sollte an einen habituellen Husten gedacht werden, früher auch als ticartiger oder psychogener Husten bezeichnet. Häufig sei er vor allem bei Jungs im Alter von 7-10 Jahren und typischerweise hätten die Kindern vor allem in $\mathrm{Ge}$ genwart von Eltern oder Lehrern extrem störenden Husten. Es gibt keine somatische Ursache, kann aber über Monate gehen, so Seidenberg. Hustenmedikamente seien in der Regel nicht wirksam. Durch Aufklärung über „eine schlechte Angewohnheit“ oder einen möglichen Erinnerungshusten nach einem Infekt seien aber oft innerhalb kurzer Zeit Erfolge zu erzielen.

Als weitere Ursachen eines chronischen Hustens nannte Seidenberg eine Bronchitis fibroplastica, die zu produktivem Husten mit Fibrinfäden führt, Husten in Verbindung mit Thoraxschmerzen, die auf eine Arrhythmie, Embolie, eine Pneumonie oder eine Pleuritis hindeuten können, sowie angeborene Herzfehler mit begleitenden Gefäßanomalien oder Herzinsuffizienz.

Roland Fath

Referenzen: 112. Jahrestagung der Deutschen Gesellschaft für Kinder- und Jugendmedizin, Hamburg, 14.-17. September 2016

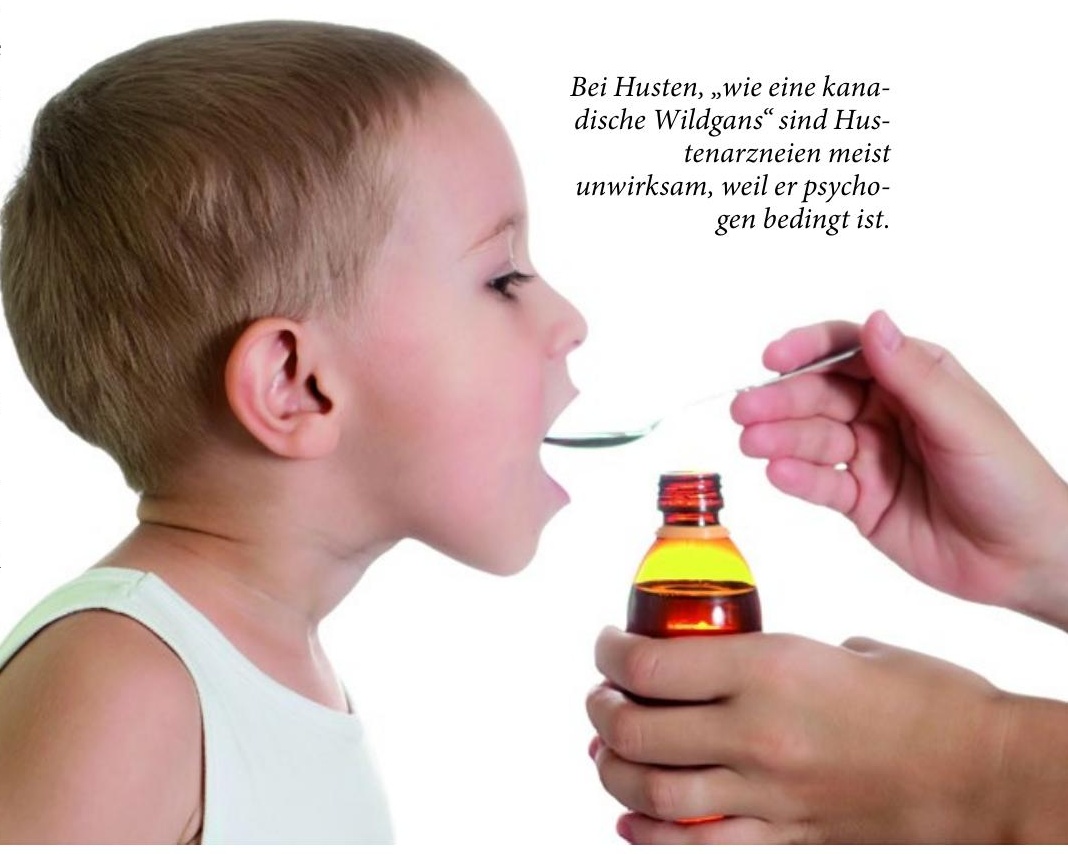

